\title{
Climate changes and suitability for the ticks Amblyomma hebraeum and Amblyomma variegatum (Ixodidae) in Zimbabwe (1974-1999)
}

\author{
Agustín Estrada-Penã, Ivan G. Horak, Trevor Petney \\ ${ }^{a}$ Faculty of Veterinary Medicine, University of Zaragoza, Miguel Servet 177, 50013- \\ Zaragoza, Spain \\ ${ }^{b}$ Faculty of Veterinary Science, University of Pretoria, Onderstepoort 0110, South Africa \\ ${ }^{c}$ Department of Zoology and Entomology, University of the Free State, Bloemfontein 9300, \\ South Africa \\ d Zoologisches Institute, Abteilung für Ökologie und Parasitologie, Universität Karlsruhe, \\ Germany
}

With 1 table and 7 figures

\begin{abstract}
The spread of $A$ mblyomma hebraeum has been reported in Zimbabwe. At the same time there was little or no spread in the distribution of $A$ mblyomma variegatum. This paper examines the climatic cycles and their trends in the period 1974-1999 with a view to explaining the abiotic causes of this spread, and of forecasting the likely tendency in climate suitability for both tick species. An annual data-set of rainfall and air temperature was used as a source for climate, together with a habitatmodeling algorithmto estimate climate suitability for both ticks. Long-term suitable habitat for $A$. hebraeum exists mainly in the south and southeast of the country. Areas of adequate habitat for $A$. variegatum exist across the country, between approximately $17^{\circ} \mathrm{S}$ and $18.5^{\circ} \mathrm{S}$, and are most suitable in regions of the northwest. The climate niches of the two species differ, and account for their almost allopatric distributions, as observed in the duration and intensity of the dry period and in total annual rainfall. Cyclic changes in both temperature and rainfall drive the periodic modifications in the distributions of the ticks. More intense periods of drought in the highveld, drive the expansion of $A$. hebraeum in this region. Temperature does not have any effect on the tendency in this area. Areas in south and southeast show a trend towards an increase in climatic suitability because of an increase in temperature. Zones in which habitat suitability is increasing for $A$. variegatum are restricted to the northwestern parts of the country, because warmer temperatures and a slight decrease in the intensity of the dry season. The progressive increase in temperatures seems to be forcing the dispersion of $A$. variegatum towards areas outside of zones that have a prolonged dry period. On the other hand $A$. hebraeum is compelled to spread northwards, following areas with adequate rainfall patterns, but halted by temperature limits and perhaps competition with $A$. variegatum. Without adequate control measures, invasive waves of $A$. hebraeum may occur over a background swell of northward expansion.
\end{abstract}

Keywords: Amblyomma hebraeum; Amblyomma variegatum; Zimbabwe; Climate cycles; Habitat suitability; Invasive events

\section{Introduction}

The tick vectors of heartwater (Ehrlichia ruminantium infection) in Zimbabwe, namely Amblyomma hebraeum and Amblyomma variegatum, were presumed to be confined to the low-lying regions of the south and of the northwest of the country, respectively. The geographical divide that separated the southern limits of $A$. variegatum from the northern limits of $A$. hebraeum was the central plateau (highveld) of Zimbabwe, on which neither species had become permanently established in stockfarming areas during the 20th century (Norval, 1983). However, countrywide surveys in 19751980, 1988- 1991 and 1996 demonstrated that both species were established in parts of the western highveld plateau.

Because of the pre-independence war, cattle dipping in the communal farming areas in the low-lying southern part of the country (southern lowveld) was disrupted between 1977 and 1980 and $A$. hebraeum, and with it heartwater, spread rapidly (Norval, 1979, 1983; Lawrence et al., 1980). By 1980 $A$. hebraeum had been recorded throughout the southern low-veld, and its distribution in central Zimbabwe extended northwards onto and beyond the highveld plateau. During this time there was some overlap between the distributions of $A$. hebraeum and $A$. variegatum. Between 1981 and 1985, when intensive dipping recommenced in the communal lands, $A$. hebraeum apparently disappeared 
from the highveld areas to which it had spread. However, it subsequently became re-established on the highveld. A. variegatum was already established in the northern lowland and there was no evidence of significant dispersion by this species. The spread of $A$. hebraeum was at the time attributed both to the breakdown in government-provided dipping services in small-holder communal farming areas, and to the movements of wild ungulates that act as alternative hosts for Amblyomma species (Peter et al., 1998b). The long-term changes in climate in the different regions of the country and how these might have affected the climate suitability for the ticks were, however, not studied in detail.

Habitat suitability modeling is an effective tool for providing innovative insights into the ecology and distribution of parasitic arthropods and the diseases they may transmit. It is also an exercise by which the expected changes in tick distribution under different climate scenarios may be explained and understood. Species distribution models are empirical models that relate field observations to environmental predictor variables based on statistically or theoretically derived response surfaces (Guisan and Zimmermann, 2000). Environmental predictors can exert direct or indirect effects on species, and in the case of ticks, it is currently recognized that climate is the main driving variable (Cumming, 2002), while vegetation and habitat patchiness play secondary roles in delineating tick distribution at the scale of landscape (Estrada-Penã, 2005).

The purpose of this study is to determine the factors that regulate the distribution of $A$. hebraeum and $A$. variegatum in Zimbabwe, to gain further knowledge about the climatic niches used by each species, as well as the trends in climate suitability for both ticks in this country.

\section{Material and methods}

\subsection{Source of tick data}

This paper is based on a compilation of records for both $A$. hebraeum and $A$. variegatum in Zimbabwe. The main body of data was provided by Peter et al. (1998a). This has been updated with adequate georeferenced records obtained from the tick collections of the University of Pretoria and the Onderstepoort Veterinary Institute, South Africa. A total of 995 A. hebraeum and 149 A. variegatum records have been included in the study. Records in the region span from 1975 to 2005.

\subsection{Source of climatic data}

We used monthly surfaces of total annual rainfall $(R)$ and mean air temperature $(T)$ obtained from a Climate Research Unit (CRU) time series data-set (CRU TS 2.0, http://www.cru.uea.ac.uk). This is a coherent, homogeneous, gridded data-set at a resolution of 0.58 covering the whole world. While this set of climate data spans the period 1901-1999, reliable records for the ticks in Zimbabwe only exist since the 1970s. Because of these discrepancies, the time frame selected for this study spans the period from 1974 to 1999.

While CRU TS 2.0 may be a comprehensive tool to assess climatic trends, its resolution is too coarse for the purposes of this study, and the database was consequently downscaled. $T$ and $R$ values for each month of the study period were calculated for pairs of coordinates blanketing the country, and then interpolated to $4 \mathrm{~km}$ using the method by Hay and Lennon (1999). This consists of a composite digital elevation model (DEM) with variable selection and thin-plate spline algorithm. Spatial coordinate data included in the multiple regression procedure comprised latitude, longitude and altitude coordinates, which were forced to be present in each of the regression models for interpolation. Monthly downscaled $T$ and $R$ variables were used for climate modeling.

\subsection{Climate matching models and computation of climate suitability}

The basic concept underlying species occurrence modeling is the definition of the climate niche: each species occurs within specific ranges of environmental variables, enabling individuals to survive and reproduce (Austin, 2002). Species occurrence can be predicted through appropriate environmental variables using habitat suitability (HS) models (Guisan and Thuiller, 2005). In inductive HS models, the relationships are generalized from a sample of observations in which species presence is matched with specific values of the environmental variables. We used MaxEnt, which allows for a modelling algorithm based on presence-only data (Phillips et al., 2004). This algorithm produces predictions from incomplete information by estimating the most uniform distribution of points of occurrence across the area of study. The evaluation of performance of the models is also carried out by MaxEnt, which determines the significance of each individual climate variable and provides a measure of the accuracy of the model. This procedure allows for the selection of the final set of significant variables. $\mathrm{HS}$, ranging from 0 to 100 , was computed separately for both species for each year during the period 1974-1999. We calculated the area under the curve (AUC) of a receiver operating characteristic 
(ROC) plot of sensitivity against (1-specificity) (Swets, 1988) as a measure of model performance. Sensitivity is defined as the proportion of true positives correctly predicted, whereas specificity is the proportion of true negatives predicted correctly. We obtained AUC data from a customized function in S-plus software.

A grid of hexagons with a radius of $6 \mathrm{~km}$ covering the whole country was created to handle yearly HS data and to define zones according to their behavior during the period of study. Three parameters define each hexagon: (1) mean HS for each species is above or below the average for the region with positive HS, (2) variability in HS is greater or smaller than $20 \%$ in the whole series of values for that hexagon and (3) there is a trend towards an increasing or decreasing HS, after smoothing of the HS values by Fourier series. Each hexagon was classified using Fuzzy logic procedures according to its mean value, variability and trend in HS. Fuzzy logic is a generalization of Boolean logic; it can manage truth-values between "completely true" and "completely false". Therefore, each hexagon was provided with a value between 0 and 100, according to its membership in the first category (HS above or below) and its membership in the second category (variability high or low) and its membership in the third category (increasing or decreasing trend). A complete zonal classification of Zimbabwe can be obtained by this procedure and each hexagon has a "membership value" according to its attributes in the period 1974-1999 (see Table 1).

\subsection{Calculation of climate niche for each species}

Calculation of the basic climate niche for each species is an adequate framework to understand the climate-derived drivers of climate suitability. A basic set of stress indices were published by Norval et al. (1994), and Petney et al. (1987) compiled a review of the ecological preferences of the two species. However, the climate niche should be understood as the whole set of conditions with which the ticks are associated. Consequently, we have adhered to the approach of Robertson et al. (2001) using the decomposition in the principal components of monthly climate variables to obtain a multidimensional niche of climate for both species. Monthly temperature and rainfall values for each year were subjected to principal components analysis (PCA). Three components explained more than $97 \%$ of the variance in the 24 climate variables, and consequently these three were retained. Axis 1 is heavily loaded with temperature (higher values represent higher temperatures), axis 2 is strongly related to rainfall seasonality (positive values are related to an intense dry season in April-August, while negative ones are correlated with a short or even missing dry period in these months). Axis 3 is related to total annual rainfall.

\section{Results}

Maps depicting average HS for both Amblyomma species in Zimbabwe during the period 1974-1999 are included as Figs. 1 and 2. The best model selected the temperature from March to September (both species), and rainfall from March to September ( $A$. hebraeum), or February to October $(A$. variegatum), as indicators of choice for model building. The AUC for the models is $0.95(A$. hebraeum) and 0.92 (A. variegatum). Longterm suitable habitat for $A$. hebraeum is present mainly in the lowveld in the south and southeast of the country. The northern limits for this species extend across Zimbabwe at approximately $19^{\circ} \mathrm{S}$. The distribution of adequate habitat for $A$. variegatum stretches across the country, between approximately $17^{\circ} \mathrm{S}$ and $18.5^{\circ} \mathrm{S}$. The highest HS for this tick is present in areas in the northwest of the country and in isolated pockets in the east. The southern half of the country and parts of the northern region appear to have no adequate HS for this species.

Climate niche as detected in the reduced space of principal components is distinctly different for both species (Fig. 3). The range of temperatures for optimum habitat is narrower for $A$. hebraeum. It also prefers drier environments and localities with a marked seasonal drought. $A$. variegatum finds higher $\mathrm{HS}$ in regions without prolonged seasonal droughts and with a higher annual rainfall. In summary, areas suitable for $A$. hebraeum experience a marked seasonal drought in March-August and tend to be slightly colder and clearly drier than those that suit $A$. variegatum. Conspicuous differences are evident in the ratio total rainfall/winter rainfall, with both species occupying distinctly different climate niches (Fig. 3E and F).

We divided Zimbabwe into zones according to the average, year-to-year variability in, and trend of HS for each species. Six zones were recognized for $A$. hebraeum (see Fig. 4 and Table 1). Zone 1 (high $\mathrm{HS}$ with a trend to increase) and 2 (high HS with a trend to decrease) occupy most of the south and southeast of Zimbabwe (Fig. 4A and B), while zone 3 has small foci in some central and eastern regions (Fig. $4 \mathrm{C}$ ). Zone 4 represents those areas where average $\mathrm{HS}$ for $A$. hebraeumis low and with a clear trend to decrease, and is restricted to north and northwest Zimbabwe (Fig. 4D). Zone 5, located mainly in the highveld, corresponds to the sites where habitat suitability for the tick has slowly been increasing since 1974 (Fig. 4E). Zone 6 consists of sites where the HS for $A$. hebraeum remains low, with high year-to-year variability, and with a tendency towards further decrease (Fig. 4F). As a whole, 
zones where the HS for $A$. hebraeum had increased between 1974 and 1999 comprise more than $25 \%$ of the total surface area of the country, while those in which HS had been decreasing comprise about $7 \%$. Approximately $41 \%$ of the surface area is composed of areas with low $\mathrm{HS}$ and a trend to further decrease (Table 1).

Only four zones were detected relative to changes in HS for A. variegatum (Fig. 5) and we have retained the same terminology for them as for $A$. hebraeum. Zones 1 and 2, which represent areas in which the tick has a high HS with a tendency to increase (zone 1), or decrease (zone 2), are focal and confined to portions of northern Zimbabwe. Zone 5, representing sites with low HS and a trend to increase, is restricted to the northeast of the country. Sites with a high HS for $A$. variegatum and a clear tendency to increase comprise only $14 \%$ of the surface area of Zimbabwe.

These zones are a product of the general climate and their trends to increase or decrease in HS during the period of study. In the case of $A$. hebraeum (Fig. 6) localities classified as belonging to zone 1 are present in areas where temperature is increasing, but with few changes in their rainfall pattern, whereas areas belonging to zone 2 are located in regions in which both temperature and seasonal drought increased throughout the period of study. HS patterns in zone 3 correspond with areas of higher total rainfall and seasonal drought, with small changes in temperature. In contrast, zone 4 is characterized by high total rainfall and temperature, as well as the absence of winter drought. The climate of zone 4 is close to the optimal for $A$. hebraeum only for short periods of time, resulting in temporary peaks of suitability. The main characteristic of the climate in zone 5 is a high total rainfall with a tendency towards prolonged periods of drought in winter. The features of zone 6 are difficult to define, as it had a low HS for the species throughout the study period, its total area is small and there is no clear trend in climate traits.

Both temperature and winter drought play a pivotal role in the definition of HS zones for $A$. variegatum (Fig. 7). Zone 1 occupies localities with a tendency towards temperature increase and a slight decrease in the intensity of the dry season, while zone 2 exhibits a trend towards an increase of intensity of the dry season. Zone 3 is colder and in the lower limit of the preference range of the species, and zone 4 is characterized by an intense dry season.

\section{Discussion}

At least two reviews concerning the ecological preferences of $A$. hebraeum and $A$. variegatum have already been published (Walker and Olwage, 1987; Petney et al., 1987), and the historical occurrence of heartwater in Zimbabwe has been described in detail (Norval et al., 1994). Subsequent studies (Peter et al., 1998b) recorded outbreaks of heartwater as well as collections of $A$. hebraeum in the highveld plateau between 1987 and 1992, a zone which is cooler and has a higher annual rainfall than the rest of the country. The baseline data for the present investigation on the distribution of $A$. hebraeum and $A$. variegatum in Zimbabwe were provided by the study conducted by Peter et al. (1998a), who made nearly 3,000 collections throughout the country during a period of 1 year. Their findings also confirmed previous observations on the northerly expansion of the distribution range of $A$. hebraeum and the easterly and westerly extension of that of $A$. variegatum. Field-collected data support the model-derived results provided in the current study.

There is no total consensus about the reliability of climate as recorded through remote sensing or by spatial interpolation of the data provided by ground recording stations (Hay and Lennon, 1999). Adequate remotely sensed information is not available for the whole time period covered in this study, and we therefore chose to use the CRU data-set of interpolated climate. The usefulness of this dataset had previously been established during studies on malaria (Small et al., 2003), and on climate preferences for ticks (Estrada-Penã and Venzal, 2006). Habitat models are useful tools for a variety of different purposes involving the ecology of parasites. It has been concluded that, on average, climatic variables are better predictors of tick distribution than vegetation-derived variables (Cumming, 2002) and that their distributions are not primarily limited by those of their hosts (Cumming, 1999). Thus, the primary factor governing tick distribution is the direct effect of climate. Preliminary attempts tomodel climate suitability for $A$. hebraeumand $A$. variegatum were performed using CLIMEX (Norval et al., 1994). These CLIMEX-derived distributions were, however, almost the opposite of the actual distributions of the ticks in Zimbabwe. This result could be interpreted as either that the variables used in the CLIMEX model are not a good predictor of the distributions of these species, or that factors other than climate affect the occurrence of the ticks. The authors suggested that the lack of fit was a consequence of the role played by non-climatic factors. The lack of adequate parameters to run the models was, however, probably the source of the mismatch, as Norval et al. (1994) concluded that the two species have similar climatic requirements and occur in similar habitats, whereas our results point towards very different climate niches used by each species. The present results obtained using a 
PCA-derived climate niche, show distinct differences in the rainfall requirements (yearly and for the period March-August) of both species that are responsible of their almost allopatric distribution. This decomposition of the redundant variables into a PCA reduced space is very valuable in defining the portions of the n-dimensional climate niche (Hirzel et al., 2001) occupied by clusters of taxa, and emerges as a very adequate tool with which to interpret the interspecific differences in climate preferences.

The results of the present study show good agreement between actual field records and modelled spatial range. The lack of coincidence that might be noticed by visual inspection of the maps, mainly for the range of $A$. hebraeum, emanates from the use of averaged long-term climate (1974-1999), while some field records represent short-term invasions. The dramatic spread of $A$. hebraeum during short-time periods has previously been reported (Norval, 1983). Models developed here confirm that the bulk of the distribution of both tick species remained relatively stable during the period 1974-1999, with a clear bias to expand for $A$. hebraeum and a smaller spatial increase in suitable climate for $A$. variegatum. These results are also in agreement with field observations suggesting that the range of adequate climate for $A$. hebraeum clearly increased in the period 1974-1999, while the long-term trend in climate provided only marginal new habitat for $A$. variegatum (Peter et al., 1998a). Drastic changes in climate may switch an area from unsuitable to suitable within a few years, and be responsible for the invasive waves reported by other authors regarding the circumstances surrounding the spread of $A$. hebraeum.

The extent of the climatically suitable areas for $A$. hebraeum in Zimbabwe fluctuated considerably during the period 1974-1999. No single climatic factor, but a combination of temperatures, total rainfall and intensity of the dry season interacted to favour periods of high $\mathrm{HS}$ for $A$. hebraeum in areas where it was previously unknown. Increased cattle and wildlife movements, and a reduced intensity of dipping undoubtedly played important roles, favouring areas with enhanced suitability for the tick that could then be invaded. While the barrier effect of dipping does not form part of this discussion, it does not explain the lack of similar expansion by $A$. variegatum in the same period, as further spread could not be accomplished without an adequate HS for the tick. Regions in which $A$. hebraeum is well established and HS is increasing have a tendency towards a well-defined and intense dry season in April-August. The absence of $A$. hebraeum from the highveld of Zimbabwe has been attributed to high rainfall or the absence of large wild ungulate species, which serve as alternative hosts, and the low ambient temperatures (Norval, 1977). The present findings indicate that the HS for A. hebraeum in this zone is increasing, mainly as a result of a long-term trend towards a more intense and longer period of drought during winter. The changes in HS for $A$. variegatum in Zimbabwe have been more limited and static than those for $A$. hebraeum. Increasing HS for this species is present only in certain small areas scattered throughout the country, in which temperatures are increasing, rainfall remains relatively high and the dry season is not severe. The HS for this tick is decreasing in areas where the dry season is becoming more acute and with only marginal changes in temperature.

Norval et al. (1994) used the changes in the approximate distribution of $A$. hebraeum and $A$. variegatum in Zimbabwe between 1975/76, 1977/78 and 1979/80 to model the rate at which the species could be expected to spread, fitting various functions to the three data points. However, the observed changes in climate are of a cyclic rather than of a linear nature. While the trends in climatic patterns can provide an insight into the expected HS in the near future, it is risky to speculate about the details, as they are of a very dynamic nature. The slow but progressive increase in temperature is driving the expansion of $A$. variegatum towards areas within its climate niche, excluding it from zones with a prolonged dry period. At the same time, $A$. hebraeum is compelled to spread northwards, following areas with adequate rainfall patterns, and halted by the limit of temperatures and perhaps competition with $A$. variegatum in areas where they coexist (Norval, 1983). However, sudden and drastic changes in climate patterns are expected to occur, producing dramatic changes in HS for short periods of time. Without adequate control measures, invasive waves of $A$. hebraeum may take place over a background of northward spreading.

\section{Acknowledgements}

The authors want to acknowledge the collaboration of Heloise Heyne in providing data from the tick collection at the Onderstepoort Veterinary Institute (South Africa). The compilation of this article has been facilitated through The Integrated Consortium on Ticks and Tickborne Diseases (ICTTD-3) financed by the International Cooperation Programme of the European Union through Coordination Action Project no. 510561. 


\section{References}

1. Austin, M.P., 2002. Spatial prediction of species distribution: an interface between ecological theory and statistical modelling. Ecol. Model. 157, 101-118.

2. Cumming, G.S., 1999. Host distributions do not limit the species range of most African ticks (Acari: Ixodida). Bull. Entomol. Res. 8, 303-327.

3. Cumming, G.S., 2002. Comparing climate and vegetation as limiting factors for species range of African ticks. Ecology 83, 225-268.

4. Estrada-Penã, A., 2005. Effects of habitat suitability and landscape patterns on tick (Acarina) metapopulation processes. Landscape Ecol. 20, 529-541.

5. Estrada-Penã, A., Venzal, J.M., 2006. Changes in habitat suitability for the tick Ixodes ricinus (Acari: Ixodidae) in Europe (1900-1999). Ecohealth 3, 154-162.

6. Guisan, A., Thuiller, W., 2005. Predicting species distribution: offering more than simple habitat models. Ecol. Lett. 8, 993-1009.

7. Guisan, A., Zimmermann, N.E., 2000. Predictive habitat distribution models in ecology. Ecol. Model. 135, 147-186.

8. Hay, S.I., Lennon, J.J., 1999. Deriving meteorological variables across Africa for the study and control of vector-borne disease: a comparison of remote sensing and spatial interpolation of climate. Trop. Med. Int. Health 4, 58-71.

9. Hirzel, A.H., Helfer, V., Metral, F., 2001. Assessing habitat-suitability models with a virtual species. Ecol. Model. 145, 111-121.

10. Lawrence, J.A., Foggin, C.M., Norval, R.A.I., 1980. The effects of war on the control of diseases of livestock in Rhodesia (Zimbabwe). Vet. Rec. 107, 82-85.

11. Norval, R.A.I., 1977. Studies on the ecology of the tick Amblyomma hebraeum Koch in the Eastern Cape province of South Africa II. Survival and development. J. Parasitol. 63, 740747.

12. Norval, R.A.I., 1979. Tick infestations and tick-borne diseases in Zimbabwe Rhodesia. J. S. Afr. Vet. Assoc. 50, 289-292.

13. Norval, R.A.I., 1983. Ticks of Zimbabwe VII: The genus Amblyomma. Zimbabwe Vet. J. 23, 115.

14. Norval, R.A.I., Perry, B.D., Meltzer, M.I., Kruska, R.L., Booth, T.H., 1994. Factors affecting the distributions of the ticks Amblyomma hebraeum and A. variegatum in Zimbabwe: implications of reduced acaricide usage. Exp. Appl. Acarol. 18, 383-407.

15. Peter, T., Perry, B.D., O'Callaghan, C.J., Medley, G.F., Shumba, W., Madzima, W., Burridge, M.J., Mahan, S.M., 1998a. Distributions of the vectors of heartwater, Amblyomma hebraeum and Amblyomma variegatum (Acari: Ixodidae), in Zimababwe. Exp. Appl. Acarol. 22, 725-740

16. Peter, T., Perry, B.D., O'Callaghan, C.J., Medley, G.F., Shumba, W., Madzima, W., Burridge, M.J., Mahan, S.M., 1998b. The distribution of heartwater in the highveld of Zimbabwe 19801997 Onderstepoort J. Vet. Res. 65, 177-187.

17. Petney, T.N., Horak, I.G., Rechav, Y., 1987. The ecology of the African vectors of heartwater, with particular reference to Amblyomma hebraeum and Amblyomma variegatum. Onderstepoort J. Vet. Res. 54, 381-395.

18. Phillips, S.E., Dudı'k, M., Shapire, R.E., 2004. A maximum entropy approach to species distribution modelling. In: Proceedings of the 21st International Conference on Machine Learning, Banff, Canada, pp. 13-19.

19. Robertson, M.P., Caithness, N., Villet, M.H., 2001. A PCA-based modelling technique for predicting environmental suitability for organisms from presence records. Div. Distrib. 7, 1527.

20. Small, J., Goetz, S.J., Hay, S.I., 2003. Climatic suitability for malaria transmission in Africa 1911-1995. Proc. Natl. Acad. Sci. 23, 15341-15345.

21. Swets, J.A., 1988. Measuring the accuracy of diagnostic systems. Science 240, 1285-1293.

22. Walker, J.B., Olwage, A., 1987. The tick vectors of Cowdria ruminantium (Ixodoidea, Ixodidae, genus Amblyomma) and their distribution. Onderstepoort J. Vet. Res. 54, 353-379. 


\section{Table 1}

Zones recognized in Zimbabwe according to the average variability and trend of habitat suitability (HS) for Amblyomma hebraeum and Amblyomma variegatum as percent of cells of the hexagonal grid overlying the country

\begin{tabular}{|c|c|c|c|c|c|c|}
\hline Species & Zone & Av HS & Var HS & Trend HS & $\%$ & Definition \\
\hline \multirow[t]{6}{*}{ A. hebraeum } & 1 & + & + & + & 17.4 & $\begin{array}{l}\text { Areas with HS above average, high variability } \\
\text { and a trend to an increase in HS }\end{array}$ \\
\hline & 2 & + & + & - & 7.6 & $\begin{array}{l}\text { Areas with HS above average, high variability } \\
\text { and a trend to a decrease in HS }\end{array}$ \\
\hline & 3 & + & - & + & 1.2 & $\begin{array}{l}\text { Areas with HS above average, low variability } \\
\text { and a trend to an increase in HS }\end{array}$ \\
\hline & 4 & - & - & - & 33.9 & $\begin{array}{l}\text { Areas with HS below average, low variability } \\
\text { and a trend to a decrease in HS }\end{array}$ \\
\hline & 5 & - & - & + & 7.3 & $\begin{array}{l}\text { Areas with HS below average, low variability } \\
\text { and a trend to an increase in HS }\end{array}$ \\
\hline & 6 & - & + & - & 4.1 & $\begin{array}{l}\text { Areas with HS below average, high variability } \\
\text { and a trend to an increase in HS }\end{array}$ \\
\hline \multirow{4}{*}{ A. variegatum } & & + & + & + & 14.1 & \\
\hline & 2 & + & + & _- & 15.1 & \\
\hline & 4 & - & - & - & 8.1 & \\
\hline & 5 & - & - & + & 5.1 & \\
\hline
\end{tabular}

See Figs. 3 and 4 for the geographical representation of these zones (av = average HS; var = variability in $\mathrm{HS}$ in a given locality). Signs + or - mean for increase or decrease. Definitions of zones for $A$. variegatum are the same and not repeated.

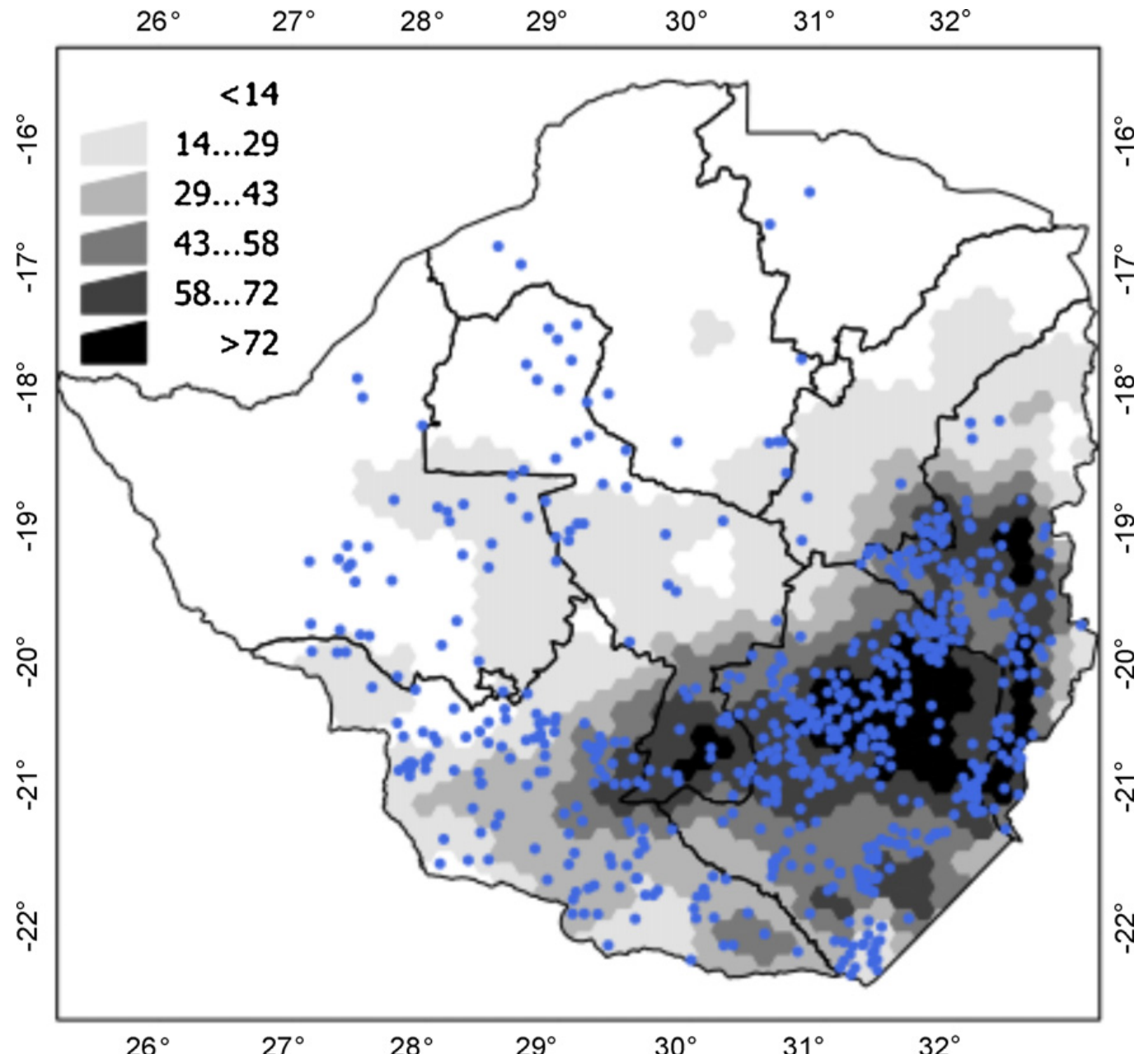

Fig. 1. Mean habitat suitability for Amblyomma hebraeum in Zimbabwe during the period 1974-1999 together with the actual records (Fig. 1dots). 


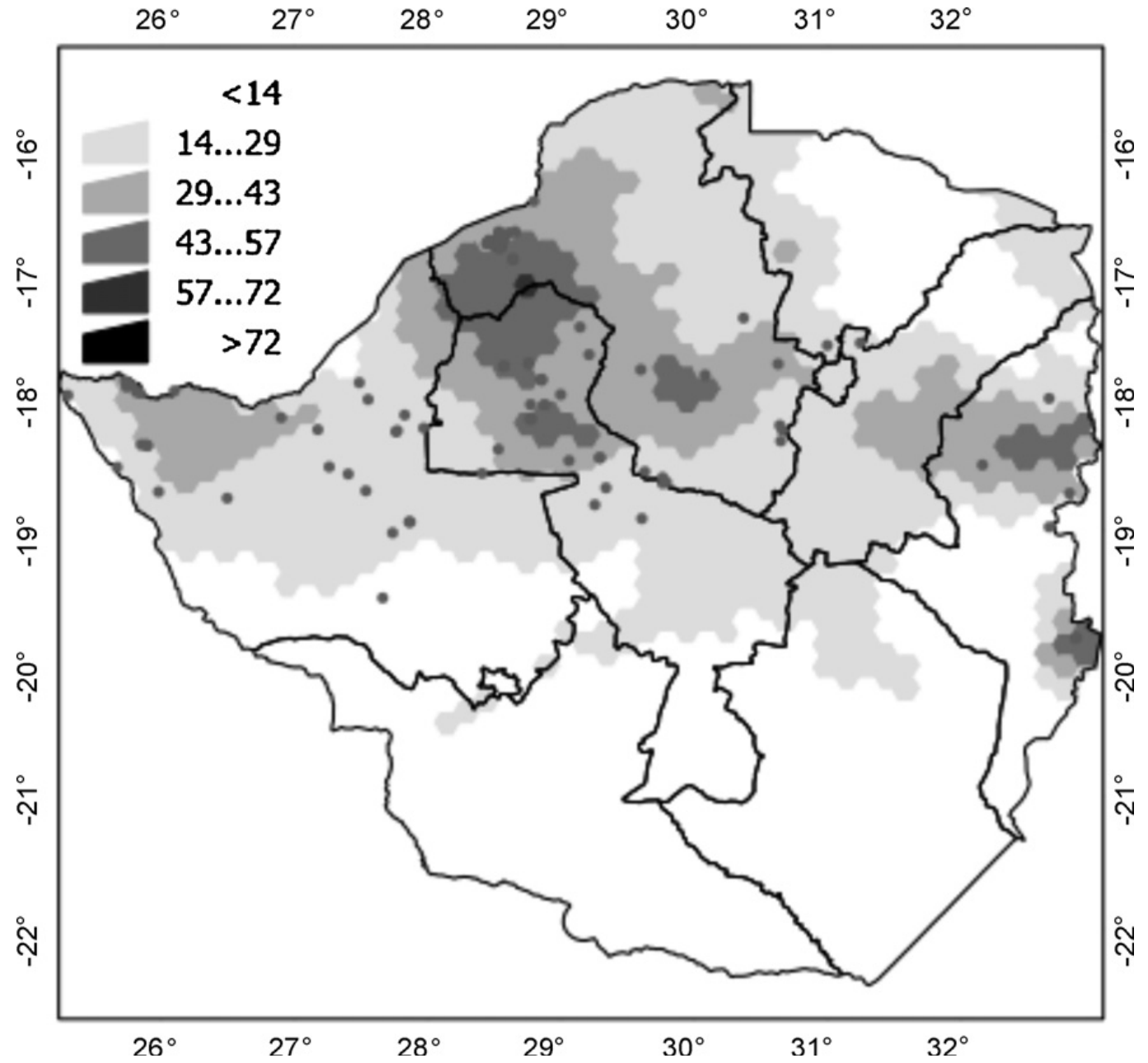

Fig. 2. Mean habitat suitability for Amblyomma variegatum in Zimbabwe during the period 1974-1999 together with the actual records (dots). 

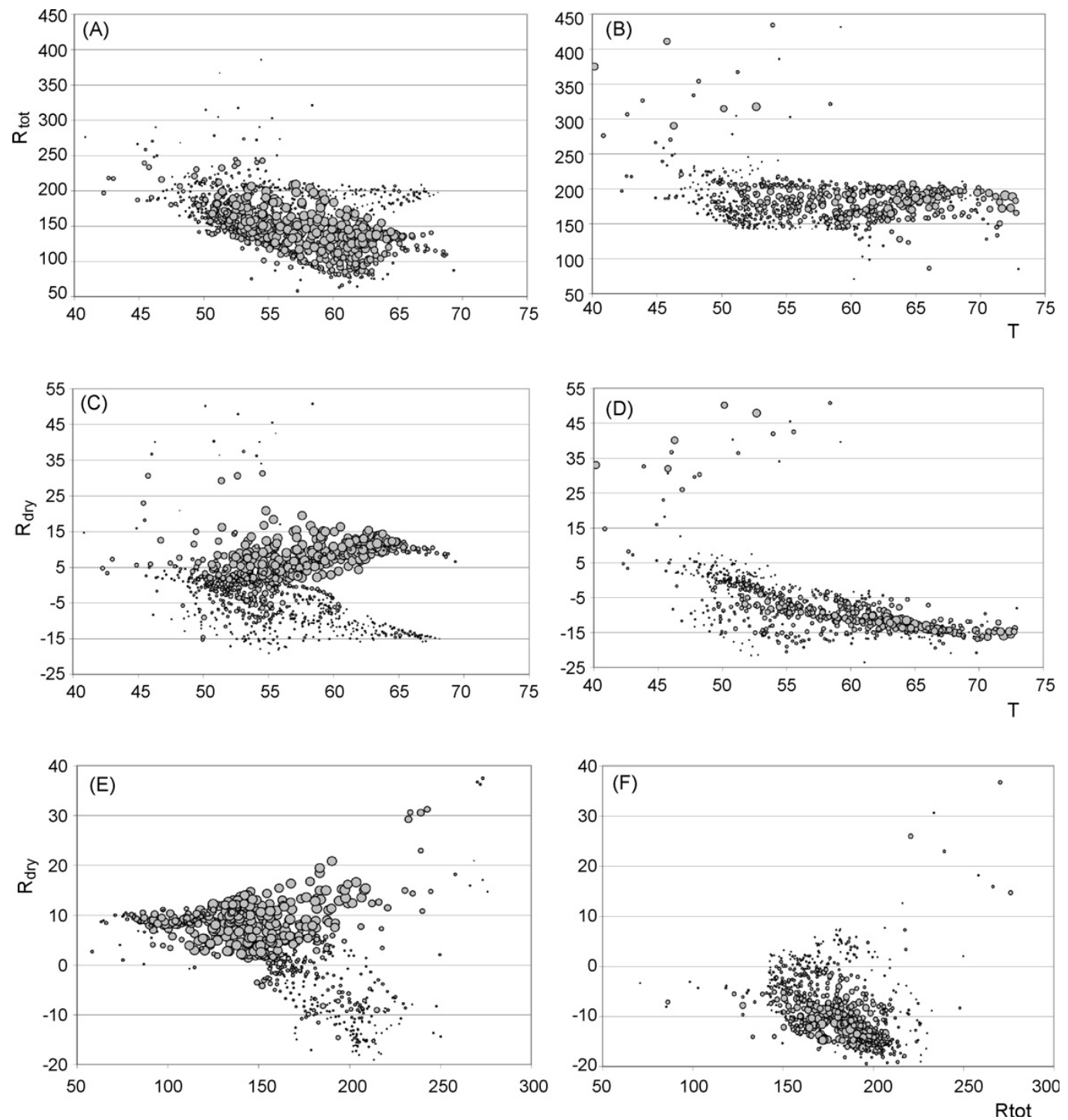

Fig. 3. Association of habitat suitability for $A$. hebraeum and $A$. variegatum with defined portions of the PCA-reduced climate niche. The diameter of dots is directly proportional to the HS. Panels $A, C$ and $E$ show the position of suitable climate for $A$. hebraeum, and panels $\mathrm{B}, \mathrm{D}$ and $\mathrm{F}$ show the same for $A$. variegatum at the same scale to emphasise the different climate requirements of both species. $R_{\text {tot: }}$ : axis depicting total annual rainfall, $\mathrm{R}_{\mathrm{dry}}$ : axis depicting rainfall in the dry season (negative numbers reflect more intense dry season); T: axis related to the total temperature in the year. Units of each axis are the values of the three retained axes in the reduced space. Dots mean for the association of positive HS of the species to any combination of climate conditions in the PCA-reduced space. 


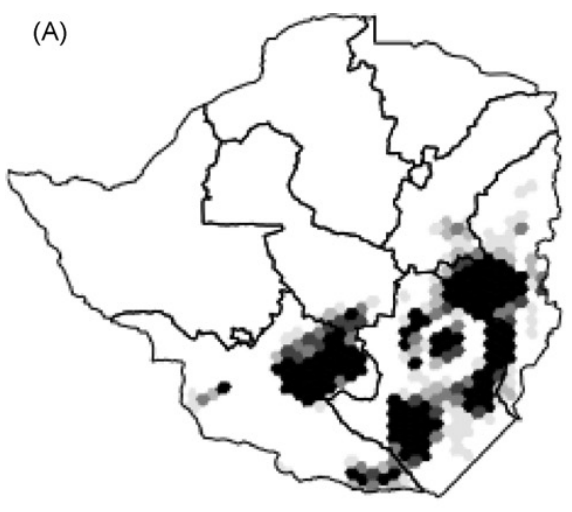

(C)

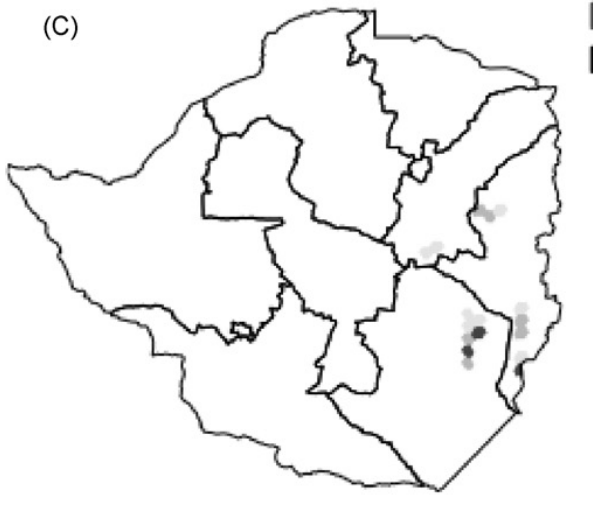

(E)

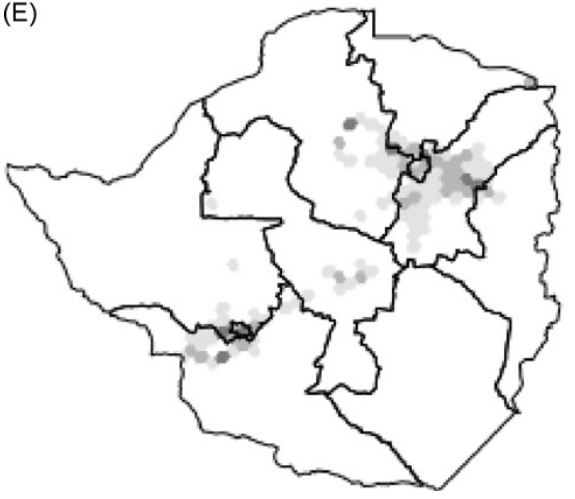

(B)

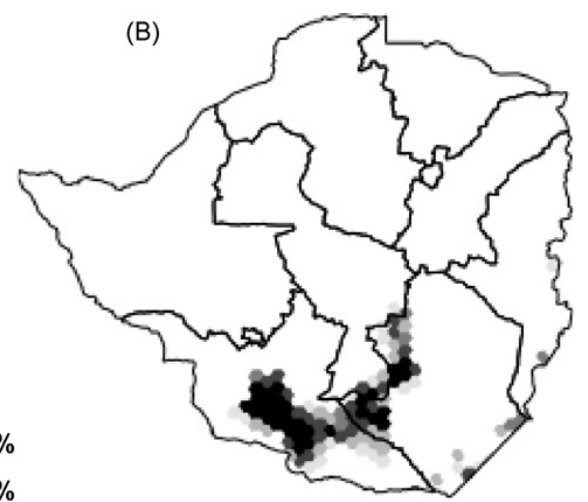

$10 \% \ldots 30 \%$

$30 \% . . .48 \%$

$66 \% . . .84 \%$

$>84 \%$
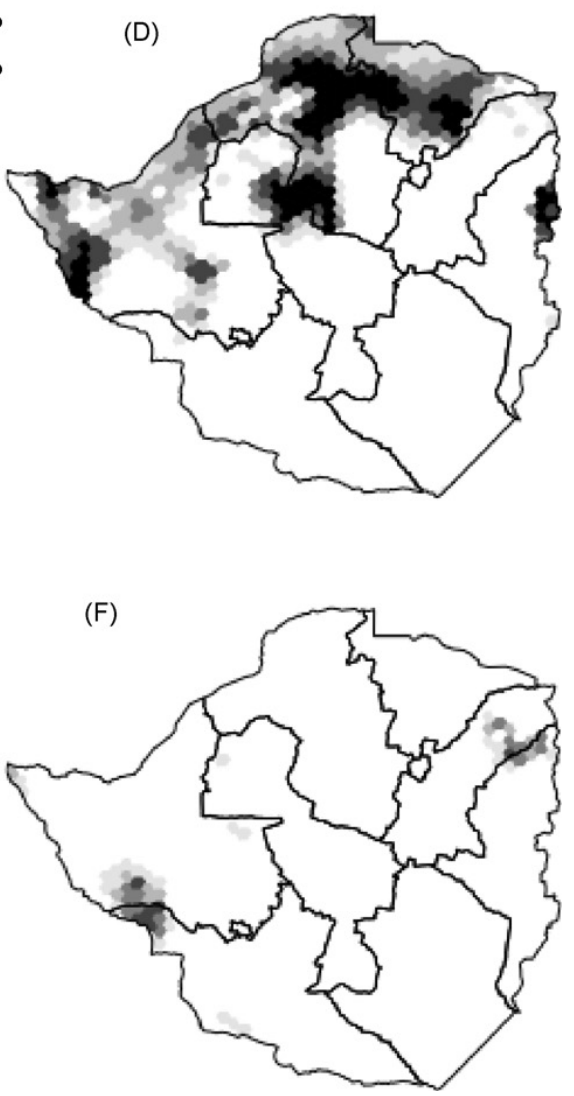

Fig. 4. Classification of Zimbabwe into zones according to their average habitat suitability (HS), their variability and trends in the period 1974-1999 for A. hebraeum. Each cell is classified according to its degree of membership of a given zone (see Table 1 for a description of the categories). For example, a darker cell in panel A denotes a stronger membership to zone 1 (high HS, high variability, trend to increase in HS). Panels A-F display membership of categories $1-6$, respectively 

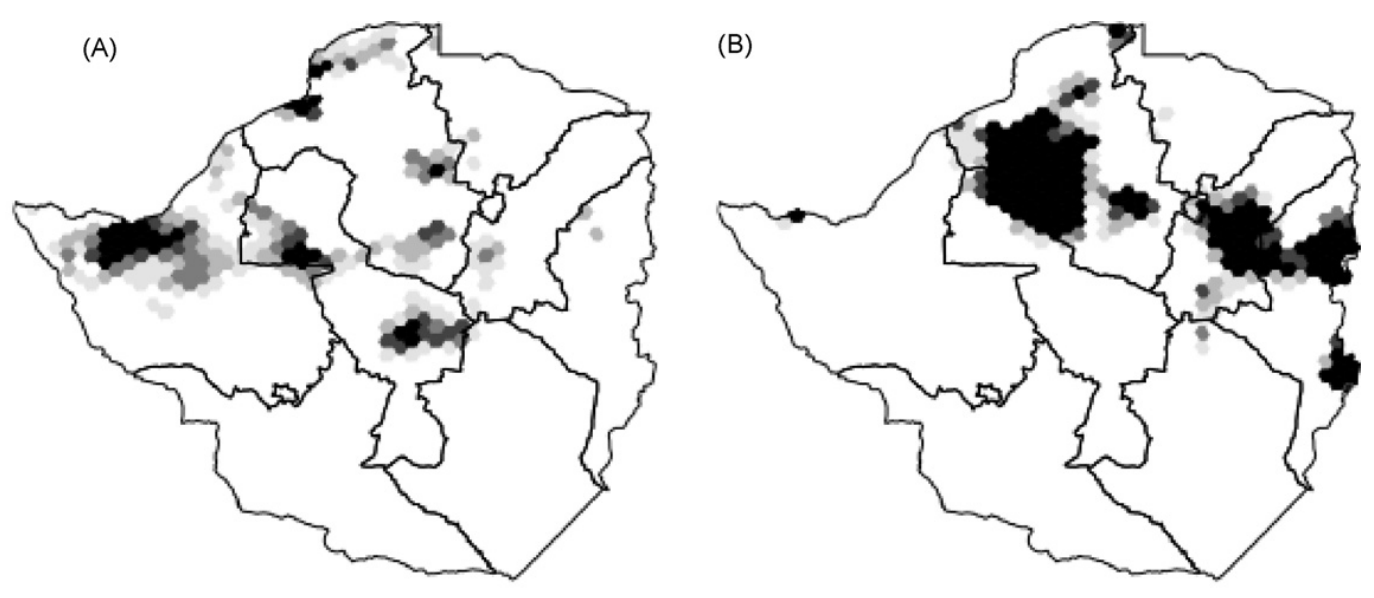

$<10 \%$

$10 \%$...30\%

$30 \% . .48 \%$

$48 \%$ \%...66 \%

$66 \% . . .84 \%$

$>84 \%$
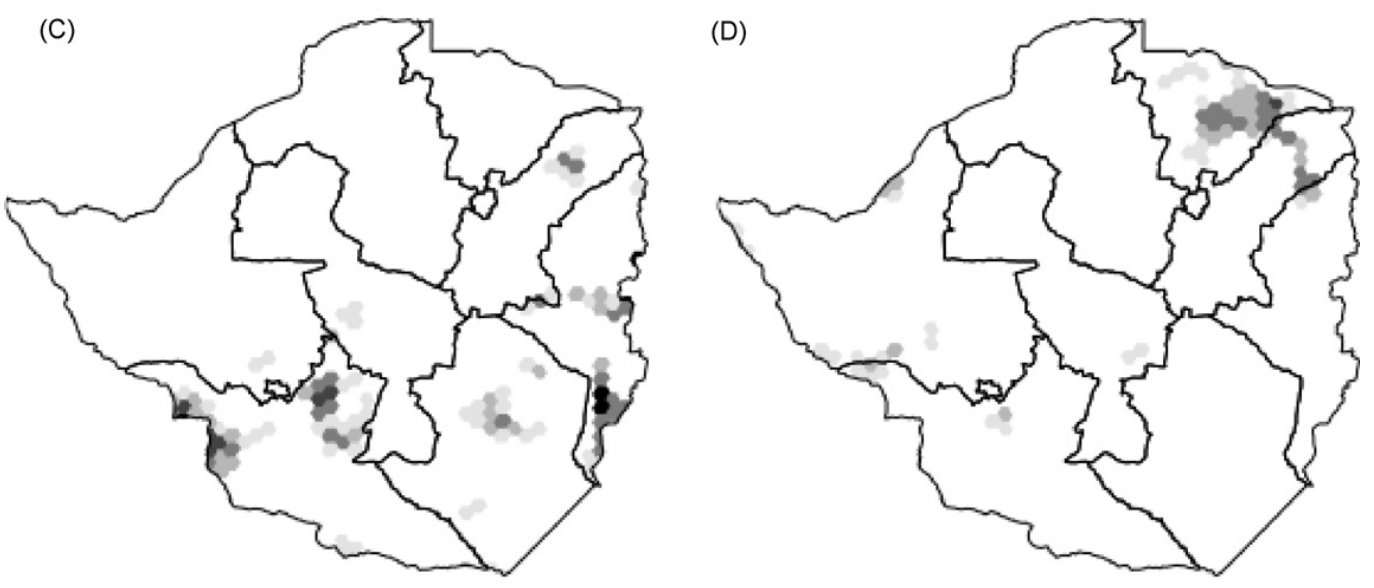

Fig. 5. Classification of Zimbabwe into zones according to their average HS, their variability and trends in the period 1974-1999 for $A$. variegatum. Each cell is classified according to its degree of membership of a given zone (see Table 1 for a description of the categories). For example, a darker cell in panel A denotes a higher membership to category 1 (high HS, high variability, trend to increasing in HS). Panels $A$ and $B$ represent categories 1 and 2, respectively; panels $C$ and $D$ represent categories 4 and 5 , respectively. 

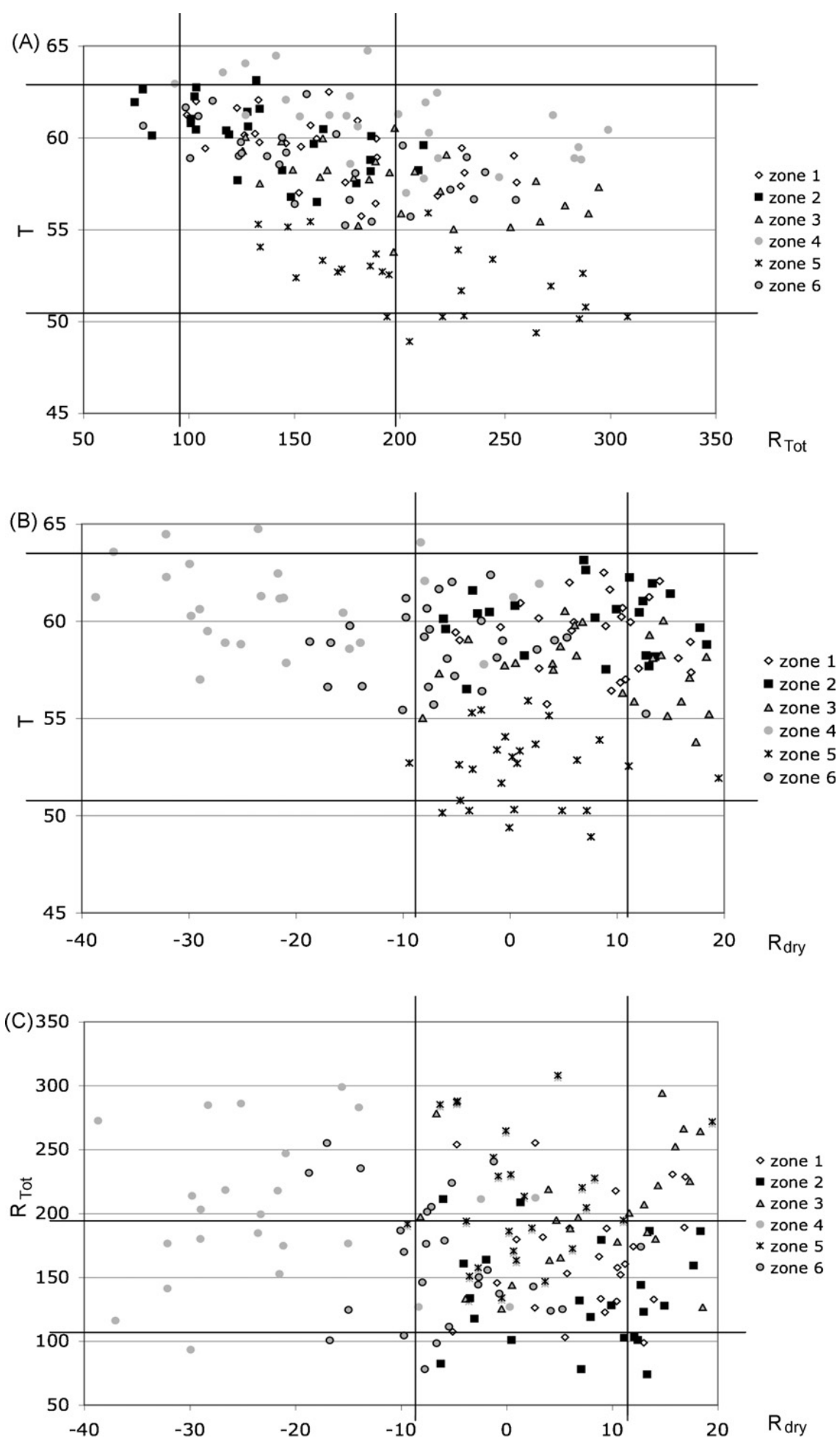

Fig. 6. Mean values of climatic factors, as extracted from the PCA axes in the time period 1974-1999 in the zones recognized in Zimbabwe in relation to their habitat suitability for $A$. hebraeum. Values are plotted against the three principal axes. $T$ : factor derived from total year temperature; $R_{\text {tot }}$ : factor derived from total annual rainfall; $R_{\text {dry }}$ : factor derived from rainfall in the dry season (negative values reflect a more intense dry season).

For simplicity, each symbol represents the mean of a factor in a given year for a zone. The two vertical and horizontal solid lines are intended to represent the long-term average conditions in the time period 1974-1999 as a square in the area of intersection. Units of axes are values of the PCA reduced space. 

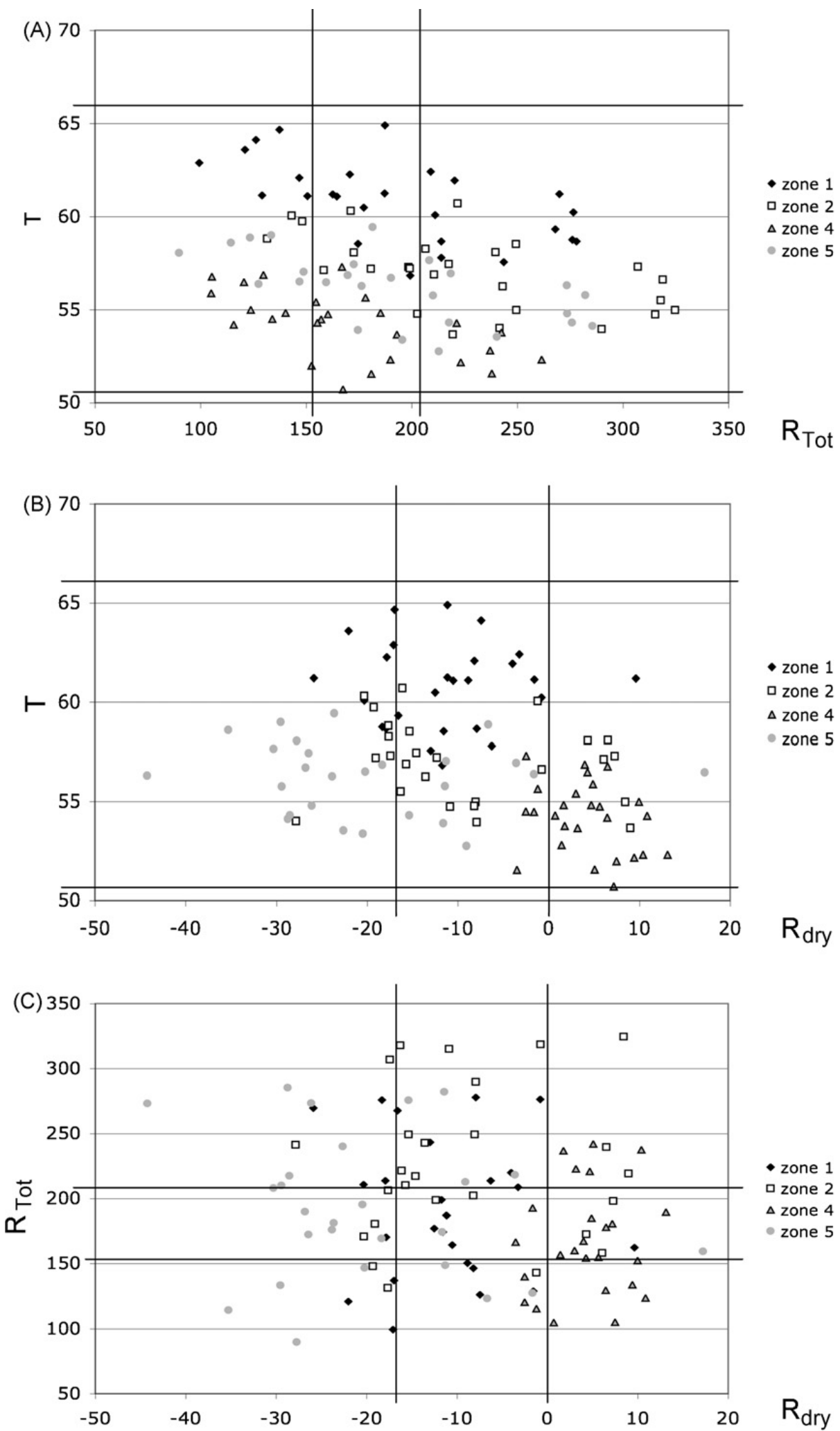

Fig. 7. Mean values of climatic factors, as extracted from the PCA axes in the period 1974-1999 in the zones recognized in Zimbabwe in relation to habitat suitability for $A$. variegatum. Values are plotted against the three principal axes. $T$ : factor derived from total year temperature; $R_{\text {tot }}$ factor derived from total annual rainfall; $R_{\text {dry: }}$ factor derived from rainfall in the dry season (negative values reflect a more intense dry season). Each symbol

represents the mean of a factor in a given year for a zone. 